Pacific

Journal of

Mathematics

ORDERING PURE BRAID GROUPS ON COMPACT, CONNECTED SURFACES

JuAn GonZÁLEZ-MENESES 


\title{
ORDERING PURE BRAID GROUPS ON COMPACT, CONNECTED SURFACES
}

\author{
JuAn GonzÁLeZ-Meneses
}

\begin{abstract}
We prove that the pure braid groups on compact, connected, orientable surfaces are bi-orderable, and that the pure braid groups on compact, connected non-orientable surfaces have generalized torsion, thus they are not bi-orderable.
\end{abstract}

\section{Introduction.}

The purpose of this paper is to answer the following question: Are pure braid groups on compact, connected surfaces bi-orderable? We will prove that the answer is positive for orientable surfaces, and negative for the non-orientable ones.

In this section we give the basic definitions and classical results. We also explain what is known about orders on braid groups, and finally we state our results. In Section 2 we study the particular case of closed, orientable surfaces. The closed, non-orientable surfaces are treated in Section 3 and, in Section 4, we extend our results to all compact, connected surfaces.

Let us just mention that, if a surface is non-connected, its braid groups are a direct product of braid groups on each connected component (it needs to be taken into acount how many base points are in each connected component). Knowing that a direct product of groups is bi-orderable if and only if each one is bi-orderable, we can extend our results to all compact surfaces.

1.1. Braids on surfaces. Let $M$ be a compact connected surface, not necessarily orientable, with or without boundary, and let $\mathcal{P}=\left\{P_{1}, \ldots, P_{n}\right\}$ be a set of $n$ distinct points in the interior of $M$. Define an $n$-braid based at $\mathcal{P}$ to be a collection $b=\left(b_{1}, \ldots, b_{n}\right)$ of disjoint smooth paths in $\operatorname{Int}(M) \times[0,1]$, called strings of $b$, such that the $i$-th string $b_{i}$ runs monotonically in $t \in[0,1]$ from the point $\left(P_{i}, 0\right)$ to some point $\left(P_{j}, 1\right), P_{j} \in \mathcal{P}$.

An isotopy is defined as a deformation through braids, which fixes the ends. Multiplication of braids is defined by concatenation. The isotopy classes of braids with this multiplication form the group $B_{n}(M, \mathcal{P})$, called braid group with $n$ strings on $M$ based at $\mathcal{P}$. Note that the group $B_{n}(M, \mathcal{P})$ does not depend, up to isomorphism, on the set $\mathcal{P}$ of points but only on the cardinality $n=|\mathcal{P}|$. So we may write $B_{n}(M)$ in place of $B_{n}(M, \mathcal{P})$. Further details can be found in $[\mathbf{B i}]$. 
A pure braid is an element $b \in B_{n}(M)$ such that $b_{i}$ ends at $\left(P_{i}, 1\right)$ for all $i=1, \ldots, n$. In other words, $b$ induces the trivial permutation on $\mathcal{P}$. Pure braids form a normal subgroup of $B_{n}(M)$ called pure braid group with $n$ strings on $M$, and denoted by $P B_{n}(M)$.

Let $D$ be a closed disc. Notice that $B_{n}=B_{n}(D)$ is the classical braid group of Artin $[\mathbf{A}]$. Recall that $B_{n}$ is generated by the set $\left\{\sigma_{1}, \ldots, \sigma_{n-1}\right\}$, where $\sigma_{i}$ is the braid shown in Figure 1.

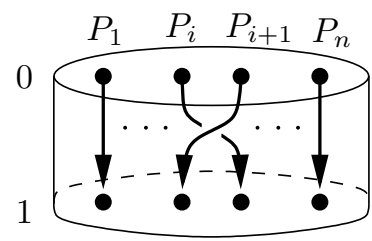

$\mathrm{D}$

Figure 1. The braid $\sigma_{i} \in B_{n}(D)$.

1.2. Orderable groups. A group $G$ is said to be right-orderable if there exists a strict total ordering $<$ on its elements which is invariant under right-multiplication: $g<h$ implies $g k<h k$ for all $g, h, k \in G$. If $<$ is also invariant under left-multiplication, then $G$ is said to be bi-orderable.

The following is a well-known characterization of right-orderable and biorderable groups.

Proposition 1.1. A group $G$ is right-orderable if and only if there exists a subset $\mathfrak{p} \subset G$ such that $\mathfrak{p}^{2} \subset \mathfrak{p}$ and $G=\mathfrak{p} \coprod\{1\} \coprod \mathfrak{p}^{-1}$. Moreover, $G$ is bi-orderable if and only if there exists such $\mathfrak{p}$ also satisfying $g \mathfrak{p} g^{-1} \subset \mathfrak{p}$, for all $g \in G$.

Proof. If $G$ is right-orderable (bi-orderable), just take $\mathfrak{p}=\{g \in G ; 1<$ $g\}$, the set of positive elements. Conversely, if there exists $\mathfrak{p}$ verifying the required hypothesis, then define the order $<$ by: $g<h$ if and only if $h g^{-1} \in$ $\mathfrak{p}$.

Let us state three properties of orderable groups, which can be found either in $[\mathbf{P}]$ or in $[\mathbf{R Z}]$.

Proposition 1.2. Let $1 \rightarrow A \stackrel{\alpha}{\rightarrow} B \stackrel{\beta}{\rightarrow} C \rightarrow 1$ be an exact sequence of groups. If $A$ and $C$ are right-orderable, with sets of positive elements $\mathfrak{p}_{A}$ and $\mathfrak{p}_{C}$ respectively, then the set $\mathfrak{p}_{B}=\alpha\left(\mathfrak{p}_{A}\right) \cup \beta^{-1}\left(\mathfrak{p}_{C}\right)$ defines a right order on $B$. Moreover, if $A$ and $C$ are bi-orderable, the order on $B$ defined in such a way is a bi-order if and only if $\alpha\left(\mathfrak{p}_{A}\right)$ is normal in $B$ (that is, if the order on $A$ is preserved by conjugation in $B)$. 
Definition 1.3. We say that a group $G$ has generalized torsion if there exist $g, h_{1}, \ldots, h_{k} \in G, g \neq 1$, such that $\left(h_{1} g h_{1}^{-1}\right)\left(h_{2} g h_{2}^{-1}\right) \cdots\left(h_{k} g h_{k}^{-1}\right)=1$.

Proposition 1.4. A bi-orderable group has no generalized torsion (in particular, it is torsion-free).

Proposition 1.5. Let $G$ be a right-orderable group, and let $R$ be a ring with no zero divisors. Then the group ring $R G$ has no zero divisors. Moreover, the only units of $R G$ are the monomials $r g$, with $r$ invertible in $R$.

1.3. Ordering braid groups. Artin braid groups $B_{n}=B_{n}(D)$ are known to be right-orderable ([D], see also $[$ FGRRW $]$ ). However they are not biorderable if $n \geq 3$, since they have generalized torsion $[\mathbf{N}]$. Nevertheless, $P_{n}=P B_{n}(D)$ is bi-orderable, as shown in $[\mathbf{R Z}]$ and in $[\mathbf{K R}]$.

In more generality, it is shown in $[\mathbf{R W}]$ (see also $[\mathbf{S W}]$ ) that the mapping class groups of compact surfaces with boundary, fixing a finite number of points, are right-orderable. Braid groups on compact surfaces with boundary are subgroups of these mapping class groups, thus they are also right-orderable.

On the other hand, it is not known if the braid groups on closed surfaces are right-orderable or not. If $M$ is a closed surface different from the sphere and from the projective plane, then $B_{n}(D) \subset B_{n}(M)$ (see $[\mathbf{P R}]$ ). Hence, $B_{n}(M)$ also has generalized torsion, and in conclusion, it cannot be biorderable.

In this paper, we will study the pure braid groups of compact connected surfaces. We treat first the case of closed surfaces, and then we extend our results to all compact connected surfaces. More precisely, we will show the following three results.

Theorem 1.6. If $M$ is a closed, orientable surface, then $P B_{n}(M)$ is biorderable.

Theorem 1.7. If $M$ is a closed, non-orientable surface, then $P B_{n}(M)$ has generalized torsion, for $n \geq 2$. Therefore, $P B_{n}(M)$ is not bi-orderable for $n \geq 2$.

Corollary 1.8. If $M$ is a compact connected surface, and $n \geq 2$, then $P B_{n}(M)$ is bi-orderable if and only if $M$ is orientable.

\section{Closed, orientable surfaces.}

In this section we prove Theorem 1.6. In Subsection 2.1 we state that free groups and fundamental groups of orientable surfaces are bi-orderable. In the case of free groups, we will explicitly define a bi-order. Then we see in Subsection 2.2 that $P B_{n}(M)$ is an extension of two groups, $K_{n}$ and $\pi_{1}(M)^{n}$, which are both bi-orderable. Moreover, the hypothesis of Proposition 1.2 are satisfied, so $P B_{n}(M)$ turns out to be bi-orderable. 
2.1. Bi-order of free groups and fundamental groups. We will explicitly define a bi-order on a given free group using the so-called Magnus expansion [MKS]. Let $F$ be a free group with free system of generators $G=\left\{x_{i}\right\}_{i \in I}$ ( $I$ not necessarily finite). Let $\mathbb{Z} \llbracket X_{I} \rrbracket$ be the ring of formal power series over the non-commutative indeterminates $\left\{X_{i}\right\}_{i \in I}$. The Magnus expansion of $F$ is a multiplicative homomorphism $M: F \rightarrow \mathbb{Z} \llbracket X_{I} \rrbracket$, such that $M\left(x_{i}\right)=1+X_{i}$ and $M\left(x_{i}^{-1}\right)=1-X_{i}+X_{i}^{2}-\cdots$, for all $i \in I$. $M$ is known to be a well-defined and injective homomorphism, whose image is contained in

$$
\mathcal{F}=\left\{1+\eta \in \mathbb{Z} \llbracket X_{I} \rrbracket ; \eta(0)=0, \eta \text { involves only finitely many variables }\right\} .
$$

Let us define a total order on $\mathcal{F}$. First, we choose a total order on the set $\left\{X_{i}\right\}_{i \in I}$. Then, we order the monomials of $\mathbb{Z} \llbracket X_{I} \rrbracket$ as follows:

$$
m_{1}<m_{2} \Leftrightarrow\left\{\begin{array}{l}
\operatorname{deg}\left(m_{1}\right)<\operatorname{deg}\left(m_{2}\right) \\
\operatorname{deg}\left(m_{1}\right)=\operatorname{deg}\left(m_{2}\right) \text { and } m_{1}<\text { lex } m_{2},
\end{array}\right.
$$

where deg means total degree (the sum of all exponents) and $<_{\text {lex }}$ means smaller in the lexicographical order. The total order $\prec$ on $\mathcal{F}$ is defined as follows: Given $f, g \in \mathcal{F}$, we say that $f \prec g$ if and only if the coefficient of the smallest non-trivial term of $g-f$ is positive (this smallest term exists since the elements of $\mathcal{F}$ involve only finitely many variables, hence they have only finitely many terms of a given degree).

Turning back to $F$, we define the Magnus order on it: Given $a, b \in F$, we say that $a<b$ if and only if $M(a) \prec M(b)$. The Magnus order is known to be a bi-order on $F$. Moreover, one has:

Theorem 2.1 ([KR]). The Magnus order on $F$ is preserved under any $\Phi \in$ $\operatorname{Aut}(F)$ which induces the identity on $H_{1}(F)=F /[F, F]$.

Let $\psi$ be a permutation of the set $\left\{X_{i}\right\}_{i \in I}$, and consider its extension $\Psi \in \operatorname{Aut}(F)$. One has:

Theorem 2.2. If $\psi$ preserves the order on $\left\{X_{i}\right\}_{i \in I}$, then $\Psi$ preserves the Magnus order on $F$.

Proof. Notice that, under the action of such a $\Psi$, the degree and the lexicographical order on the monomials are preserved. Hence, $\Psi$ preserves the order we defined on the monomials, thus the order $\prec$ on $\mathcal{F}$. Therefore, the Magnus order on $F$ is also preserved.

We finish this subsection with the following result.

Theorem 2.3 ([Ba]). If $M$ is a closed, orientable surface, then $\pi_{1}(M)$ is a bi-orderable group. 
2.2. $\boldsymbol{P} \boldsymbol{B}_{\boldsymbol{n}}(\boldsymbol{M})$ is bi-orderable. Let $M$ be a closed, orientable surface. Given a pure braid $b=\left(b_{1}, \ldots, b_{n}\right) \in P B_{n}(M)$, we can consider, for all $i=1, \ldots, n$, the loop $\mu_{i}$ in $M$ constructed as follows: Take the $i$-th string $b_{i}$ (which is a path in $M \times[0,1]) ; \mu_{i}$ is its projection over the first coordinate (i.e., over $M)$. Since $b \in P B_{n}(M), \mu_{i}$ is a loop in $M$ based at $P_{i}$ for all $i=1, \ldots, n$, which represents an element of $\pi_{1}\left(M, P_{i}\right) \cong \pi_{1}(M)$. This defines an epimorphism $\theta: P B_{n}(M) \rightarrow \pi_{1}(M)^{n}$, which sends $\left(b_{1}, \ldots, b_{n}\right)$ to $\left(\mu_{1}, \ldots, \mu_{n}\right)($ see $[\mathbf{B i}])$.

Define $K_{n}=\operatorname{ker}(\theta)$. One has the exact sequence

$$
1 \longrightarrow K_{n} \longrightarrow P B_{n}(M) \stackrel{\theta}{\longrightarrow} \pi_{1}(M)^{n} \longrightarrow 1 \text {. }
$$
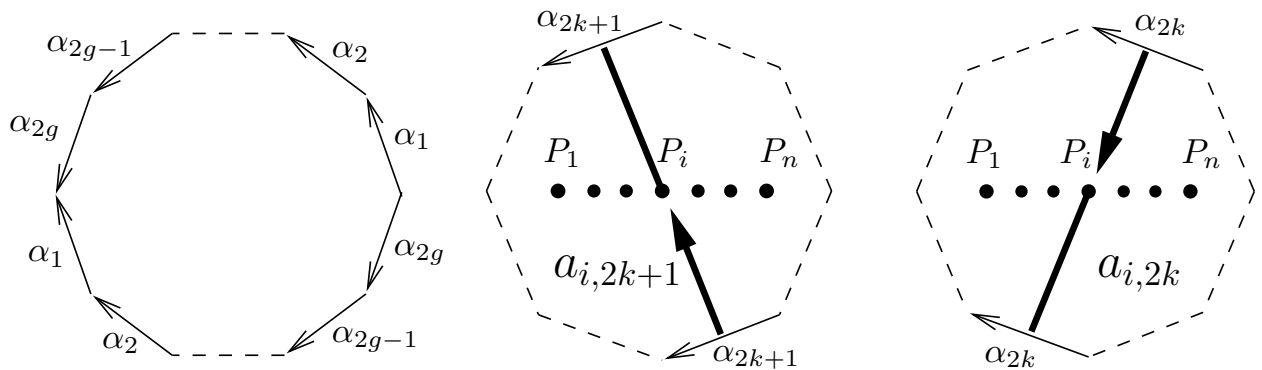

Figure 2. The polygon representing $M$ and the braids $a_{i, 2 k+1}$ and $a_{i, 2 k}$.

Let us prove that $K_{n}$ is bi-orderable. First, we represent $M$ as a polygon of $4 \mathrm{~g}$ sides, identified in the way of Figure 2. For all $i=1, \ldots, n$ and all $r=1, \ldots, 2 g$, we define the braid $a_{i, r} \in P B_{n}(M)$ as in Figure 2: The $i$-th string of $a_{i, r}$ is $\left(s_{i, r}(t), t\right) \in M \times[0,1]$, where $s_{i, r}$ is a loop in $M$ based at $P_{i}$ which goes through the wall $\alpha_{r}$; it goes upwards if $r$ is odd and downwards if $r$ is even. The $j$-th string of $a_{i, r}$ is $\left(P_{j}, t\right)$ (the trivial string) for all $j \neq i$.

Let now $\Omega=\left\{\omega_{1}, \ldots, \omega_{2 g}\right\}$ be a set of generators of $\pi_{1}(M)$, where $g$ is the genus of $M$. Take $\Omega$ in such a way that

$$
\pi_{1}(M)=\left\langle\Omega ; \omega_{1} \cdots \omega_{2 g} \omega_{1}^{-1} \cdots \omega_{2 g}^{-1}=1\right\rangle .
$$

For all $\gamma \in \pi_{1}(M)$, choose a unique word $\widetilde{\gamma}$ over $\Omega \cup \Omega^{-1}$ representing $\gamma$. We denote by $\widetilde{\gamma}_{(i)}$ the pure braid obtained from $\widetilde{\gamma}$ by replacing $\omega_{r}^{ \pm 1}$ with $a_{i, r}^{ \pm 1}$. Now, for all $i, j \in\{1, \ldots, n\}, i<j$, we define the braid

$$
t_{i, j}=t_{j, i}=\sigma_{i} \cdots \sigma_{j-2} \sigma_{j-1}^{2} \sigma_{j-2}^{-1} \cdots \sigma_{i}^{-1} \in P B_{n}(M) .
$$

Finally, for all $i, j \in\{1, \ldots, n\}, i \neq j$, and all $\gamma \in \pi_{1}(M)$, we define $f_{i, j, \gamma}=$ $\widetilde{\gamma}_{(i)} t_{i, j} \widetilde{\gamma}_{(i)}^{-1}$. 
Theorem $2.4([\mathbf{G}-\mathbf{M P}])$. One has $K_{n}=\left(F_{n} \rtimes\left(F_{n-1} \rtimes\left(\cdots\left(F_{3} \rtimes F_{2}\right) \cdots\right)\right)\right)$, where for all $i=1, \ldots, n-1, F_{(n+1)-i}$ is the free group freely generated by $\mathcal{F}_{i, n}=\left\{f_{i, j, \gamma} ; i<j \leq n, \gamma \in \pi_{1}(M)\right\}$. Moreover, for all $m=2, \ldots, n-1$, $K_{m}=\left(F_{m} \rtimes\left(\cdots\left(F_{3} \rtimes F_{2}\right) \cdots\right)\right)$ acts trivially on $H_{1}\left(F_{m+1}\right)$.

Corollary 2.5. $K_{n}$ is bi-orderable.

Proof. We argue by induction on $n$. If $n=2$, then $K_{n}=F_{2}$ is a free group (of infinite rank), so it is bi-orderable. Suppose then that $n>2$, and that $K_{n-1}$ is bi-orderable. By Theorem 2.4, we have an exact sequence

$$
1 \longrightarrow F_{n} \longrightarrow K_{n} \longrightarrow K_{n-1} \longrightarrow 1,
$$

where $K_{n}=F_{n} \rtimes K_{n-1}$. By definition of bi-order, conjugation by an element of $F_{n}$ is an automorphism of $F_{n}$ which preserves the Magnus order. We also know, by Theorem 2.4, that conjugation by an element of $K_{n-1}$ is an automorphism of $F_{n}$ which is trivial on $H_{1}\left(F_{n}\right)$. Hence, by Theorem 2.1, it also preserves the Magnus order on $F_{n}$. Therefore, conjugation by an element of $K_{n}$ preserves the Magnus order of $F_{n}$ and thus, by Proposition 1.2, $K_{n}$ is bi-orderable.

Let us define an explicit bi-order on $K_{n}$. First, for all $i=1, \ldots, n-1$, we order $\mathcal{F}_{i, n}$ as follows:

$$
f_{i, j, \gamma}<f_{i, k, \delta} \Leftrightarrow\left\{\begin{array}{l}
j<k \\
j=k \text { and } \gamma<_{\pi_{1}} \delta,
\end{array}\right.
$$

where $<_{\pi_{1}}$ is a fixed bi-order of $\pi_{1}(M)$. Then, we consider the Magnus order on each $F_{(n+1)-i}$ corresponding to this order on $\mathcal{F}_{i, n}$. The bi-order on $K_{n}$ which yields from Corollary 2.5 is the following: For $k, k^{\prime} \in K_{n}$, write $k=k_{1} k_{2} \cdots k_{n-1}$ and $k^{\prime}=k_{1}^{\prime} k_{2}^{\prime} \cdots k_{n-1}^{\prime}$, where $k_{i}, k_{i}^{\prime} \in F_{(n+1)-i}$. Then $k<k^{\prime}$ if and only if $k_{j}<k_{j}^{\prime}$ for the greatest $j$ such that $k_{j} \neq k_{j}^{\prime}$.

Proof of Theorem 1.6. The direct product of bi-orderable groups is clearly bi-orderable, hence, by Theorem $2.3, \pi_{1}(M)^{n}$ is bi-orderable. So, by Proposition 1.2 , we only need to show that conjugation by an element of $P B_{n}(M)$ is an automorphism of $K_{n}$ which preserves the order.

Conjugation by an element of $K_{n}$ preserves the order by definition of biorder. Hence, it suffices to show the above claim for the conjugation by pre-images under $\theta$ of the generators of $\pi_{1}(M)^{n}$. A set of such pre-images is $\left\{a_{i, r} ; i=1, \ldots, n, r=1, \ldots, 2 g\right\}$. Now, in [G-MP, Lemma 3.15] it is shown that the following relations hold in $H_{1}\left(K_{n}\right)$ :

$$
a_{i, r} f_{j, k, \gamma} a_{i, r}^{-1} \equiv \begin{cases}f_{j, k, \gamma} & \text { if } i \neq j, k \\ f_{j, k,\left(\omega_{r} \gamma\right)} & \text { if } i=j \\ f_{j, k,\left(\gamma \omega_{r}^{-1}\right)} & \text { if } i=k\end{cases}
$$


We claim that the action of $a_{i, r}$ preserves the Magnus order on each $F_{m}$, $m=2, \ldots, n-1$, and hence, it preserves the order on $K_{n}$. Clearly, the action of $a_{i, r}$ on $K_{n}$ is the composition of an automorphism $\Psi_{i, r}$ which permutes the generators of each $F_{m}$, with an automorphism $\Phi_{i, r}$ which is trivial on $H_{1}\left(K_{n}\right)$. Therefore, by Theorems 2.1 and 2.2, it suffices to prove that the permutation induced by $\Psi_{i, r}$ on $\mathcal{F}_{j, n}(j=1, \ldots, n-1)$ preserves the defined order on $\mathcal{F}_{j, n}$.

Let then $f_{j, k, \gamma}, f_{j, l, \delta} \in \mathcal{F}_{j, n}$, where $f_{j, k, \gamma}<f_{j, l, \delta}$.

Case 1. If $k<l$, then $\Psi_{i, r}\left(f_{j, k, \gamma}\right)=f_{j, k, \gamma^{\prime}}<f_{j, l, \delta^{\prime}}=\Psi_{i, r}\left(f_{j, l, \delta}\right)$, where $\gamma^{\prime}$ and $\delta^{\prime}$ are determined by the above relations.

Case 2. If $k=l$ and $\gamma<_{\pi_{1}} \delta$, then there are three possibilities. First, if $i \neq j, k$, one has $\Psi_{i, r}\left(f_{j, k, \gamma}\right)=f_{j, k, \gamma}<f_{j, k, \delta}=\Psi_{i, r}\left(f_{j, k, \delta}\right)$. If $i=j$, one has $\Psi_{i, r}\left(f_{j, k, \gamma}\right)=f_{j, k,\left(\omega_{r} \gamma\right)}<f_{j, k,\left(\omega_{r} \delta\right)}=\Psi_{i, r}\left(f_{j, k, \delta}\right)$, since $\omega_{r} \gamma<_{\pi_{1}} \omega_{r} \delta\left(<_{\pi_{1}}\right.$ is a left-order). Finally, if $i=k$, then $\Psi_{i, r}\left(f_{j, k, \gamma}\right)=f_{j, k,\left(\gamma \omega_{r}^{-1}\right)}<f_{j, k,\left(\delta \omega_{r}^{-1}\right)}=$ $\Psi_{i, r}\left(f_{j, k, \delta}\right)$, since $\gamma \omega_{r}^{-1}<_{\pi_{1}} \delta \omega_{r}^{-1}\left(<_{\pi_{1}}\right.$ is a right-order $)$.

Therefore, $P B_{n}(M)$ is a bi-orderable group.

\section{Closed, non-orientable surfaces.}

We turn now to prove Theorem 1.7. Let $M$ be a closed, non-orientable surface, and let $\mathcal{P}=\left\{P_{1}, \ldots, P_{n}\right\} \subset M$. Then, there exists a submanifold $N, \mathcal{P} \subset N \subset M$, such that $N$ is homeomorphic to a Möbius strip.

Consider the subset $C=\mathbb{R} \times[0,1] \times[0,1] \subset \mathbb{R}^{3}$, and identify $(x, y, t) \sim$ $(x+1,1-y, t)$ for all $x \in \mathbb{R}$, and all $y, t \in[0,1]$. One has $(C / \sim) \simeq N \times[0,1]$. We choose $P_{i}=\left(1 / 2, p_{i}\right) \in N$, where $p_{i}=\frac{i}{n+1}$ for all $i=1, \ldots, n$.

Denote by $\Gamma=\left(\gamma_{1}, \ldots, \gamma_{n}\right)$ the braid on $N$ defined as follows: $\gamma_{i}(t)=$ $\left(1 / 2-t, p_{i}, t\right)$, for all $i=1, \ldots, n$. It is the braid represented in Figure 3 , for $n=4$. Notice that $\Gamma$ is not a pure braid (going through the wall reverses the orientation). Denote by $\Delta$ the following braid on $N$ : $\Delta=\left(\sigma_{1} \cdots \sigma_{n-1}\right)\left(\sigma_{1} \cdots \sigma_{n-2}\right) \cdots\left(\sigma_{1} \sigma_{2}\right) \sigma_{1}$. It is also drawn in Figure 3, for $n=4$. Remark that we represent $N \times\{0\}$ above $N \times\{1\}$ to agree with the usual orientation of braids (pointing downwards).
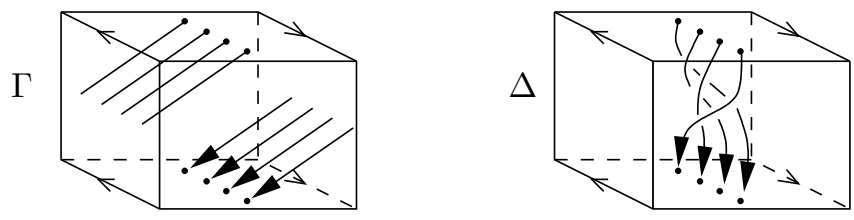

Figure 3. The braids $\Gamma, \Delta \in B_{4}(N)$.

Now, given a point $p=(x, y, t) \in C / \sim$, we denote by $\bar{p}$ the image of $p$ under reflection in the plane $y=1 / 2$. That is, $\bar{p}=(x, 1-y, t)$. In the same 
way, given a braid $b \in B_{n}(N)$, we denote by $\bar{b}$ the image of $b$ under the same reflection $\left(\bar{b}_{i}(t)=\overline{b_{i}(t)}\right)$.

Lemma 3.1. For all $b \in B_{n}(N)$, one has $\Gamma b \Gamma^{-1}=\bar{b}$.

Proof. Let $b=\left(b_{1}, \ldots, b_{n}\right) \in B_{n}(N)$. For all $i=1, \ldots, n$, we write $b_{i}(t)=$ $\left(\beta_{i}(t), t\right)$, where $\beta_{i}$ is a path on $N$. We denote by $\varepsilon$ the permutation induced by $b$ on $\mathcal{P}$. Consider the braid $c=\left(c_{1}, \ldots, c_{n}\right)=\Gamma b \Gamma^{-1}$. For all $i=1, \ldots, n$, one has

$$
c_{i}(t)= \begin{cases}\left(1 / 2-3 t, p_{i}, t\right) & \text { if } t \in[0,1 / 3], \\ \left(\beta_{n+1-i}(3 t-1), t\right) & \text { if } t \in[1 / 3,2 / 3], \\ \left(1 / 2+(3 t-2), p_{\varepsilon(n+1-i)}, t\right) & \text { if } t \in[2 / 3,1] .\end{cases}
$$

Now consider the isotopy $H:(N \times[0,1]) \times[0,1] \rightarrow N \times[0,1]$ given by

$$
H(x, y, t, s)= \begin{cases}(x+3 t s, y, t) & \text { if } t \in[0,1 / 3], \\ (x+s, y, t) & \text { if } t \in[1 / 3,2 / 3], \\ (x+3(1-t) s, y, t) & \text { if } t \in[2 / 3,1] .\end{cases}
$$

Then $H(c, 0)=c$ and $H(c, 1)=1 \bar{b} 1 \simeq \bar{b}$.

Proof of Theorem 1.7. Take a closed disc $D, \mathcal{P} \subset D \subset N \subset M$. It is well-known that, in $B_{n}(D), \Delta \sigma_{i} \Delta^{-1}=\sigma_{n-i}$ for all $i=1, \ldots, n-1$. So, the same relation holds in $B_{n}(N)$ and in $B_{n}(M)$ (every isotopy can be extended by the identity outside $D)$. Hence, for $i=1, \ldots, n-1$, one has $(\Gamma \Delta) \sigma_{i}(\Gamma \Delta)^{-1}=\Gamma \sigma_{n-i} \Gamma^{-1}=\overline{\sigma_{n-i}}=\sigma_{i}^{-1}$ in $B_{n}(N)$, thus in $B_{n}(M)$.

Therefore, one has $\sigma_{i}\left[(\Gamma \Delta) \sigma_{i}(\Gamma \Delta)^{-1}\right]=1$, and so, $\sigma_{i}^{2}\left[(\Gamma \Delta) \sigma_{i}^{2}(\Gamma \Delta)^{-1}\right]=$ 1 in $B_{n}(M)$. It suffices to notice that $\sigma_{i}^{2}$ and $\Gamma \Delta$ are pure braids, and that $\sigma_{i}^{2} \neq 1$ in $P B_{n}(M)$ for all $i=1, \ldots, n$, where $n \geq 2$, to conclude that $P B_{n}(M)$ has generalized torsion.

\section{Compact surfaces with boundary.}

Proof of Corollary 1.8. We just need to see what happens with the compact, connected surfaces with boundary.

Let $M_{g, c}$ be a surface of genus $g$ with $c$ boundary components. It is well-known that, for all $n \geq 1, B_{n}\left(M_{g, c}\right)$ is a subgroup of $B_{n+c}\left(M_{g, 0}\right)$; it suffices to consider each boundary component as a new base point which does not move. In the same way, $P B_{n}\left(M_{g, c}\right)$ is a subgroup of $P B_{n+c}\left(M_{g, 0}\right)$. Therefore, if $M_{g, c}$ is orientable, $P B_{n}\left(M_{g, c}\right)$ is bi-orderable, being a subgroup of a bi-orderable group.

On the other hand, if $M_{g, c}$ is non-orientable, there exists a submanifold $N, \mathcal{P} \subset N \subset M$, homeomorphic to a Möbius strip. Hence, if $n \geq 2$, we can apply the same technique as in the previous section, to prove that $P B_{n}\left(M_{g, c}\right)$ is not bi-orderable. 


\title{
References
}

[A] E. Artin, Theory of braids, Annals of Math., 48 (1946), 101-126, MR 8,367a, Zbl 0030.17703.

[Ba] G. Baumslag, On generalised free products, Math. Z., 78 (1962), 423-438, MR 25 \#3980, Zbl 0104.24402.

[Bi] J.S. Birman, Braids, Links and Mapping Class Groups, Annals of Math. Studies, 82, Princeton University Press, 1973, MR 54 \#13894, Zbl 0305.57013.

[D] P. Dehornoy, From large cardinals to braids via distributive algebra, J. Knot Theory Ramifications, 4(1) (1995), 33-79, MR 96g:20056, Zbl 0873.20030.

[FGRRW] R. Fenn, M.T. Greene, D. Rolfsen, C. Rourke and B. Wiest, Ordering the braid groups, Pacific J. Math., 191(1) (1999), 49-74, MR 2000j:20064.

[G-MP] J. González-Meneses and L. Paris, Vassiliev invariants of surface braid groups, Prépublication no. 218 du Laboratoire de Topologie de l'Université de Bourgogne.

[KR] D.M. Kim and D. Rolfsen, Ordering groups of pure braids and hyperplane arrangements, preprint.

[MKS] W. Magnus, A. Karras and D. Solitar, Combinatorial Group Theory. Presentations of Groups in terms of Generators and Relations, Revised ed., Dover Publications, Inc., New York, 1976, MR 54 \#10423, Zbl 0362.20023.

[N] L.P. Neuwirth, The status of some problems related to knot groups, Topology Conference (Virginia Polytech. Inst. and State Univ., Blacksburg, Va.,1973), Springer, Berlin, Lecture notes in Math., 375 (1974), 209-230, MR 55 \#4129, Zbl 0288.55003.

[P] L. Paris, On the fundamental group of the complement of a complex hyperplane arrangement, in 'Arrangements-Tokyo, 1998', Advanced Studies in Mathematics, 27, Mathematical Society of Japan, Kinokuniya, Tokyo, (2000), 257-272, MR 2001k:32050.

[PR] L. Paris and D. Rolfsen, Geometric subgroups of surface braid groups, Ann. Inst. Fourier (Grenoble), 49(2) (1999), 417-472, MR 2000f:20059, Zbl 0962.20028.

[RZ] D. Rolfsen and J. Zhu, Braids, orderings and zero divisors, J. Knot Theory Ramifications, 7(6) (1998), 837-841, MR 99g:20072, Zbl 0928.20031.

[RW] C. Rourke and B. Wiest, Order automatic mapping class groups, Pacific J. Math., 194(1) (2000), 209-227, MR 2001f:57003.

[SW] H. Short and B. Wiest, Ordering mapping class groups after Thurston, L'enseignement Math., 46 (2000), 279-312.

Received July 10, 2000 and revised February 8, 2001

\author{
UNIVERSITÉ DE BOURGOGNE \\ LABORATOIRE DE TOPOLOGIE \\ UMR 5584 DU CNRS \\ B. P. 47870 \\ 21078 - Dijon Cedex \\ FRANCE
}


Departamento de Álgebra

Facultad de Matemáticas

Universidad DE SEVILla

C/ TARFiA, S/N

41012 - SEVILLA

SPAIN

E-mail address: meneses@us.es

This author was partially supported by DGESIC-PB97-0723 and by the european network TMR Sing. Eq. Diff. et Feuill. 